\title{
Is Vitamin B12 status a risk factor for falling in older adults ( $>60 \mathrm{yrs})$ ?
}

\author{
E. Laird ${ }^{1}$, L. Hoey ${ }^{2}$, M. Healy ${ }^{3}$, M. Casey ${ }^{4}$, C. Cunningham ${ }^{4}$, W. Chong ${ }^{1}$, M. Ward ${ }^{2}$, J. J. Strain ${ }^{2}$, \\ H. McNulty ${ }^{2}$ and A. M. Molloy ${ }^{1}$ \\ ${ }^{1}$ Institute of Molecular Medicine, School of Medicine, Trinity College, Dublin, ${ }^{2}$ Northern Ireland Centre for Food and \\ Health, University of Ulster, Coleraine, ${ }^{3}$ Department of Biochemistry \& Clinical Pathology, St James's Hospital, Dublin, \\ ${ }^{4}$ Mercers Institute for Research on Ageing, St James's Hospital, Dublin
}

Currently, over $30 \%$ of older adults (>65 yrs) fall each year and $20-30 \%$ suffer from a fall-related injury ${ }^{(1)}$. Such injuries are a major public healthcare and socio-economic burden and can result in increased morbidity and fracture risk, frailty and mortality ${ }^{(1)}$. Therefore, fall prevention is a key public health issue. Previously, evidence had suggested that optimal nutritional status plays a crucial role in prevention of falls with extensive data existing for nutrients such as vitamin D and calcium ${ }^{(2)}$. However, recent evidence suggests that vitamin B12 may also play a significant role. Vitamin B12 deficiency exists within 5-40\% of the elderly population and can result in megaloblastic anaemia and demyelinating neurological disease ${ }^{(3)}$. Furthermore, concentrations of homocysteine accumulate in instances of B12 deficiency which have been linked with optic atrophy and myopia, impaired cognitive function and Alzheimer disease; conditions which are associated with increased risk of falling ${ }^{(4,5)}$. Currently, few studies have investigated markers of B12 status as a risk factor for falling in large datasets from older adult populations. Therefore, the objective of this study was to assess markers of B12 status and risk of falling in a sample of Irish older adults $(n$ 4939) from the Trinity, Ulster, Department of Agriculture (TUDA) observational study. Participants were recruited between March 2009 and May 2012 and markers of vitamin B12 status included total serum B12 (microbiological assay NCIB 12519, ATCC 43787) and Holo TC (Active B12) (Abbott AxSYM). Serum vitamin D was assessed by LCMS/MS (AB Sciex, USA) and fall related data were collected by health and lifestyle questionnaire.

Table 1. B12 status as a predictor of falling ${ }^{1}$

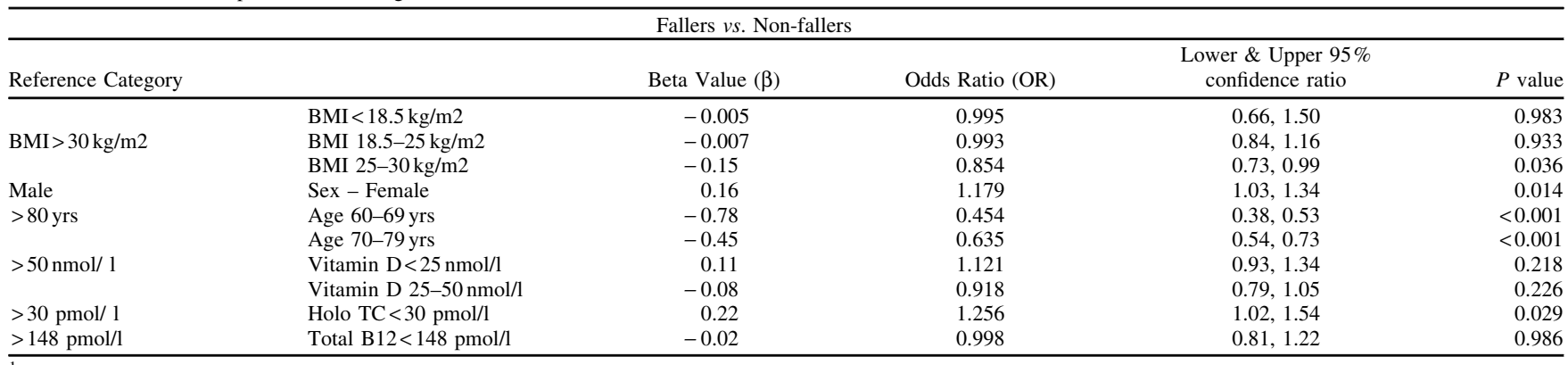

${ }^{1}$ Reference category is Non- fallers (Multi -nominal logistic regression).

A total of $1,759(36.3 \%)$ of participants had indicated they had fallen in the past year and a significant association (Partial Pearson correlation correcting for age, sex, BMI and vitamin D) was observed between Holo TC and the number of falls $(r=-0.059, P=0.028)$. A multinomial logistic regression analysis model (Table 1) indicated that a deficient B12 status (defined as Holo TC $<30$ pmol/l) was associated with an increased risk of falling $(\beta=0.22, P=0.029)$ after adjustment for other covariates.These findings demonstrate the potential importance of B12 status in the prevention of falls and further highlights the importance of addressing B12 deficiency within the older population.

This research was funded by the Irish Department of Agriculture, Fisheries and Food and the Northern Ireland Department for Employment and Learning, through its 'Strengthening the all-Island Research Base' initiative. Axis-Shield Diagnostics Ltd supported the Active B12 analysis.

1. O'Loughlin J, Robitaille Y, Boivin JF et al. (1993) Am J Epidemiol 137(3), 342-54.

2. Laird E, Ward M, McSorley E et al. (2010) Nutrients 2(7), 693-724.

3. Stabler SP, Lindenbaum J, Allen, RH et al. (1997) Am J Clin Nutr 66(4), 741-49.

4. Krumdieck CL \& Prince CW (2000) J Nutr 130(2) 365-68.

5. Gjesdal CG, Vollset SE, Ueland PM et al. (2007) Am Soc Bone Min Res 22(5), 747-756. 